\title{
Identification of gene fragments related to nitrogen deficiency in Eichhornia crassipes (Pontederiaceae)
}

\author{
Minghui $\mathrm{Fu}^{1 *}$, Lihua Jiang ${ }^{1}$, Yuanmei $\mathrm{Li}^{1}$, Guohua Yan ${ }^{1}$, Lijun Zheng ${ }^{1} \&$ Jinping Peng ${ }^{2}$ \\ 1. Department of Bioengineering, Guangdong University of Technology, Guangzhou, PR China; mhfugd@126.com \\ 2. Department of Chemistry Engineering, Guangdong University of Technology, Guangzhou, PR China; \\ 14998382@qq.com \\ * Correspondence
}

\author{
Received 20-XII-2013. Corrected 25-V-2014. Accepted 27-VI-2014.
}

\begin{abstract}
Eichhornia crassipes is an aquatic plant native to the Amazon River Basin. It has become a serious weed in freshwater habitats in rivers, lakes and reservoirs both in tropical and warm temperate areas worldwide. Some research has stated that it can be used for water phytoremediation, due to its strong assimilation of nitrogen and phosphorus, and the accumulation of heavy metals, and its growth and spread may play an important role in environmental ecology. In order to explore the molecular mechanism of E. crassipes to responses to nitrogen deficiency, we constructed forward and reversed subtracted cDNA libraries for E. crassipes roots under nitrogen deficient condition using a suppressive subtractive hybridization (SSH) method. The forward subtraction included 2100 clones, and the reversed included 2650 clones. One thousand clones were randomly selected from each library for sequencing. About 737 (527 unigenes) clones from the forward library and 757 (483 unigenes) clones from the reversed library were informative. Sequence BlastX analysis showed that there were more transporters and adenosylhomocysteinase-like proteins in E. crassipes cultured in nitrogen deficient medium; while, those cultured in nitrogen replete medium had more proteins such as UBR4-like e3 ubiquitinprotein ligase and fasciclin-like arabinogalactan protein 8-like, as well as more cytoskeletal proteins, including actin and tubulin. Cluster of Orthologous Group (COG) analysis also demonstrated that in the forward library, the most ESTs were involved in coenzyme transportation and metabolism. In the reversed library, cytoskeletal ESTs were the most abundant. Gene Ontology (GO) analysis categories demonstrated that unigenes involved in binding, cellular process and electron carrier were the most differentially expressed unigenes between the forward and reversed libraries. All these results suggest that E. crassipes can respond to different nitrogen status by efficiently regulating and controlling some transporter gene expressions, certain metabolism processes, specific signal transduction pathways and cytoskeletal construction. Rev. Biol. Trop. 62 (4): 1637-1648. Epub 2014 December 01.
\end{abstract}

Key words: Eichhornia crassipes, SSH, deficient nitrogen, BlastX analysis, COG analysis, GO analysis.

Eichhornia crassipes (Mart.) Solms. is an invasive aquatic plant that originated in South America. It is one of the most productive plants in the world, and due to its rapid reproduction, strong assimilation of nitrogen and phosphorus (Reddy, Agami, \& Tucker, 1989, 1990) and accumulation of heavy metals (Deng, Ye, \& Wong, 2004; Odjegba \& Fasidi, 2007; Caldelas, Iglesia-Turino, Araus, Bort, \& Febrero, 2009), it can be used in phytoremediation of eutrophic lakes and rivers (Wang et al., 2013), industrial waste-water (Casabianca, Laugier,
\& Posada, 1995), breeding waste-water (Lu, Fu, \& Yin, 2008; Chen, Chen, Wan, Weng, \& Huang, 2010) and landfill leachate (El-Gendy, Biswas, \& Bewtra, 2006). However, the mechanisms for its high efficiencies of absorbance and utilization of nitrogen and phosphorus remain unknown.

There have been several reports on how E. crassipes responds to different environmental conditions. Xie, Wen, Yu, and Li (2004) found E. crassipes growth was stimulated by increased nutrient concentration, and the 
plant could duly regulate biomass allocation to optimize resource acquisition in eutrophic environments. Ripley, Muller, Behenna, Whittington-Jones, and Hill (2006) also found that $E$. crassipes growth at low nitrogen and phosphorus levels could have the effect of decreasing phytosynthesis efficiency. Li, Gong, and Chang (2008) studied the effects of nitrogen form on $E$. crassipes growth and physiological responses by investigating the relative growth rate, the number of generated ramets, nitrate concentration, nitrate reductase activity, ammonium concentration, and glutamine synthetase activity. Reddy et al. (1989, 1990) evaluated the influence of phosphorus and nitrogen supply rates on growth and nutrient storage by E. crassipes. Notably, the existing studies were about morphological and physiological responses.

As far as we know, there are no reports describing the molecular response of $E$. crassipes to different nutrient conditions. In this study, we constructed forward and reversed subtraction cDNA libraries for E. crassipes under nitrogen deficient condition and identified gene fragments whose expressions were enhanced or lowered under this condition. Through the analysis of the functions of these genes, we could identify a potential molecular mechanism for E. crassipes adaption to different nutrient conditions and understand its invasion efficiency. These results also provide a theoretical basis for controlling E. crassipes growth from the nutrient metabolism perspective and could facilitate further development and utilization of E. crassipes as a polluted water remediation plant.

\section{MATERIALS AND METHODS}

Culture: Four or five $3-5 \mathrm{~cm}$ high whole plants of E. crassipes with two or three leaves were collected from the lake in the West of Guangdong University of Technology. The plant roots were washed with water, sterilized with sodium hypochlorite, and rinsed with distilled water. Finally, the plants were cultivated in a tank $\left(40 \mathrm{~cm} * 25 \mathrm{~cm}^{*} 10 \mathrm{~cm}\right)$ containing a preculture solution slightly modified according to the main components in Hoagland $\left(0.25 \mathrm{mM} \mathrm{Ca}\left(\mathrm{NO}_{3}\right)_{2} \cdot 4 \mathrm{H}_{2} \mathrm{O}, 0.25 \mathrm{mM} \mathrm{KNO}_{3}\right.$, $0.1 \mathrm{mM} \mathrm{MgSO}_{4} \cdot 7 \mathrm{H}_{2} \mathrm{O}$, and $0.05 \mathrm{mM} \mathrm{KH}_{2} \mathrm{PO}_{4}$ ) for 20 days at $28^{\circ} \mathrm{C}$, under a $12 / 12$-hour light $(20001 \mathrm{x}) /$ dark cycle. The solution was replaced every two days. After preculture, the plants were firstly cultured in maintenance culture (distilled water) for two weeks, and then cultured in experiment culture $(1.8 \mathrm{mM}$ $\mathrm{NH}_{4} \mathrm{Cl}$ solution or continued in distilled water) for 10 days.

Total RNA extraction and mRNA isolation: Total RNA of E. crassipes roots was extracted using Trizol reagent (Invitrogen, USA) according to the manufacturer's instructions. The mRNA was purified with an Oligotex mRNA Kit (Qiagen); this mRNA was precipitated with $75 \%$ alcohol, air dried and solved in Diethylpyrocarbonate DEPC treated water. Total RNA integrity was examined by electrophoresis on a $1 \%$ agarose/EtBr gel.

SSH library construction: A forward subtraction library, where the driver cDNA is subtracted from test cDNA, consists of genes that are up-regulated in the test, while a reversed subtraction library, where the test cDNA is subtracted from driver cDNA, consists of down-regulated genes (Diatchenko et al., 1996). In our experiment, E. crassipes cultured in distilled water was regarded as the test condition, and that in ammonium solution was the driver. The forward and reversed subtraction libraries were constructed with a PCR-Select cDNA Subtraction kit (Clontech) according to the manufacturer's instructions. The subtracted cDNA obtained for differentially expressed genes was cloned into the pMD18-T vector for transformation into Escherichia coli $\mathrm{DH} 5$ cells. The recombinant white clones from LB agar were randomly picked and cultured in $\mathrm{LB}$ medium at $37^{\circ} \mathrm{C}$ overnight. To evaluate subtraction efficiency, the $E$. crassipes actin gene (GenBank accession No. KC505366) was compared with subtracted and unsubtracted cDNA by quantitative real-time 
PCR analysis (qPCR). The qPCRs were carried out using actin-specific primers (Table 1). All $\mathrm{qPCR}$ reactions were performed in triplicate.

DNA sequencing and sequence data analysis: Positive white clones from each subtractive cDNA library were selected for sequencing. The sequences of clones were preprocessed to eliminate sequences of vector, adaptor, chimeric clone, pollution, repeating, and short Expressed Sequence Tag (EST) less than 100bp. Next, these informative sequences were clustered and assembled into unigenes. A unigene is a set of transcripts that appear to stem from the same transcription locus. There are two methods for clustering, supervised clustering and unsupervised clustering. Supervised clustering is that ESTs are classified with respect to known reference sequences, while unsupervised clustering is that ESTs are classified without any prior knowledge. In our experiment, we used a combination of supervised and unsupervised methods to cluster ESTs. The program Phrap (http://www. phrap.org/phredphrap/phrap.html) was used for ESTs assembly. The parameters of minmatch, minscore and repeat_stringency were set to 40, 40 and 0.96 respectively. Using NCBI BlastX and BlastP (Altschul, Gish, Miller, Myers, \& Lipman, 1990) program (http://blast.ncbi.nlm. nih.gov/Blast.cgi), all these informative ESTs were searched against the NCBI nonredundant protein sequence (NR) database and COGs database (Tatusov, Koonin, \& Lipman, 1997; Tatusov et al., 2003). The parameter e-value for searching NR database was set to $10^{-5}$ and for searching COGs database was set to $10^{-10}$. After similarity search (e-value threshold is set to $10^{-5}$ ), we used GO (http://amigo.geneontology.org/cgi-bin/amigo/blast.cgi) to classify all functions of informative ESTs and plotted GO annotations with WEGO (Ye et al., 2006) (http://wego.genomics.org.cn/cgi-bin/wego/ index.pl).

Analysis of differential ESTs by qRTPCR: For qPCR analysis, E. crassipes actin gene (GenBank accession No. KC505366)
TABLE 1

Primer sequences for PCR

\begin{tabular}{|c|c|}
\hline Primer name & Primer sequences \\
\hline actin-F1 & 5-GAGTTTGATCCTGGCTCAG-3 \\
\hline actin-R1 & 5-AGAAAGGAGGTGATCCAGCC-3 \\
\hline actin-F2 & 5-TCAATGTGCCTGCCATGTAT-3 \\
\hline Actin-R2 & 5-CCGTTCTGCAGTGGTAGTGA-3 \\
\hline 18srDNA-F & 5-GCATTTGCCAAGGATGTTTT-3 \\
\hline 18srDNA-R & 5-GCTATGTCCGGACCTGGTAA-3 \\
\hline FuM-G1-F & GTCTATAGGGCAGCGTGGTC \\
\hline FuM-G1-R & CATCTTGGCCTTCTTCTTCG \\
\hline FuM-G2-F & ATGCTCTCCACCTCATCCAG \\
\hline FuM-G2-R & AAGTTGAACTCCCAAACGTAGC \\
\hline FuM-G3-F & GGTATGTGCCGCTTTCCTAA \\
\hline FuM-G3-R & GTATACTAGGGCGCGTCGAG \\
\hline FuM-G4-F & AAACGGCTACCACATCCAAG \\
\hline FuM-G4-R & CCTCCAATGGATCCTCGTTA \\
\hline FuM-G5-F & GGTTGAGAACCAAGCCAAGA \\
\hline FuM-G5-R & AGAGTTCGAGGACGAGGACA \\
\hline FuM-G6-F & ATGTTCTCGCAGCGATCTTT \\
\hline FuM-G6-R & GATTCCTTGTTGGTGGTGCT \\
\hline FuM-G7-F & CTGGATTCCCTGATGGTGAC \\
\hline FuM-G7-R & TTCCTTTGTATCGCGAGCTT \\
\hline FuM-G8-F & TCTCAAGTGCCGAGGAAAGT \\
\hline FuM-G8-R & AACAAGGATGAGGCTGATGG \\
\hline FuM-G9-F & CACCATTGACAACGTGAAGG \\
\hline FuM-G9-R & TGAAGGGTGGATTCCTTCTG \\
\hline FuM-G10-F & ATCGGTGTTCGGAATCGTAG \\
\hline FuM-G10-R & CCGTTCCAGCATATGAACCT \\
\hline FuM-G11-F & GGCCGAGGTACAGGAACTTT \\
\hline FuM-G11-R & CACCTACGGCCTCACTCCT \\
\hline FuM-G12-F & GGCATACAGGTCCTTCCTGA \\
\hline FuM-G12-R & СТCTTCCAGCCCTCСТTCAT \\
\hline FuM-G13-F & GGCATACAGGTCCTTCCTGA \\
\hline FuM-G13-R & TCCCTGGAAAAGAGCTACGA \\
\hline FuM-G14-F & CCGCACCCTCCTTGTAGTAA \\
\hline FuM-G14-R & CTGGATGAGGGGAATCAAGA \\
\hline FuM-G15-F & CACAAACCCACCCTTGACTT \\
\hline FuM-G15-R & TGGCTTCGCCTAATGGTTAC \\
\hline FuM-G16-F & CAACTCTCGGGAAACGGTAA \\
\hline FuM-G16-R & GAAGACGTAGGGCGTTCTTG \\
\hline FuM-G17-F & TCACAGGAGACACGAGGATG \\
\hline FuM-G17-R & GCAGGCTATCTTCCTGCACA \\
\hline FuM-G18-F & TTTCGGAAGCAACAGTGACA \\
\hline FuM-G18-R & TCCAAGGCTGCTGCTTCTAC \\
\hline FuM-G19-F & AGGCTCTCACCAAGAGGACA \\
\hline FuM-G19-R & ATCAGGCACGCATTAAGACC \\
\hline FuM-G20-F & GCGGACTCCAGCTAAACAAG \\
\hline FuM-G20-R & TACAGCAGCCCTCCTTCACT \\
\hline FuM-G21-F & ТTТСТСТСТССАTGCACACG \\
\hline FuM-G21-R & GCCTCGTCCTCAACTACTGC \\
\hline
\end{tabular}


TABLE 2

Evaluation of the subtraction efficiency

\begin{tabular}{lccc} 
& $\begin{array}{c}\text { Ct value (using unsubtracted } \\
\text { cDNA as template) }\end{array}$ & $\begin{array}{c}\text { Ct value (using subtracted } \\
\text { cDNA as template) }\end{array}$ & $\begin{array}{c}\text { The differential abundance of actin in } \\
\text { unsubstracted cDNA relative to substracted cDNA }\end{array}$ \\
Forward subtraction & $19.91 \pm 0.18$ & $29.74 \pm 0.26$ & 910 times \\
Reverse subtraction & $19.56 \pm 0.28$ & $29.39 \pm 0.27$ & 910 times \\
\hline
\end{tabular}

was used as an endogenous control gene. We randomly selected 10 genes from forward library and 11 genes from the reversed library to perform qRT-PCR to evaluate the differential expression of these genes in samples cultured in nitrogen deplete and nitrogen replete media. The primers sequences for the 21 ESTs (FuMG1-F and FuM-G1-R to FuM-G21-F and FuMG21-R) and actin gene (actin-F2 and actin-R2) (listed in Table 1) were designed according to the EST and actin gene sequences. Relative gene expression levels were represented by the $2^{-\Delta \Delta \mathrm{Ct}}$ method (Livak \& Schmittgen, 2001).

\section{RESULTS}

Quality of total RNA and mRNA: Electrophoresis of the isolated total RNA on $1 \%$ agarose gels showed two bright bands corresponding to ribosomal $28 \mathrm{~s}$ and $18 \mathrm{~s}$ RNA, with an intensity ratio of 1.8-2.0:1, respectively. The purified mRNA appeared as a smear with normal dispersion (Photos not showed). These results indicated that total RNA and mRNA were intact.

\section{PCR analysis of subtraction efficiency:} The efficiency of the constructed SSH library was estimated by comparing the abundance of the housekeeping gene actin before and after subtraction. The actin gene was amplified with the templates of subtractive and unsubtractive products of the second round by qPCR. The results showed that for both forward and reversed subtraction, the differential abundance of unsubtracted and subtracted cDNA arrived at 910 times (Table 2). It indicated that a large number of constitutive genes were effectively removed in the SSH library, and the few genes with differential expression were easily detected.

Screening transformants for insertion and sequencing: In forward subtraction, a total of 2100 positive clones were identified with blue/white selection, while there were 2650

\section{A}

bp

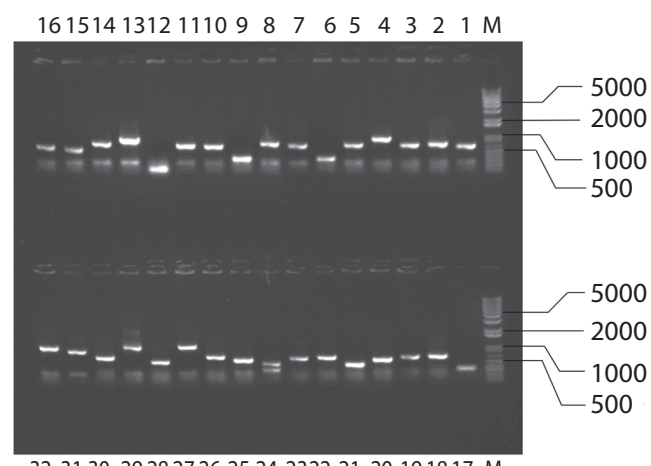

32313029282726252423222120191817 M

B

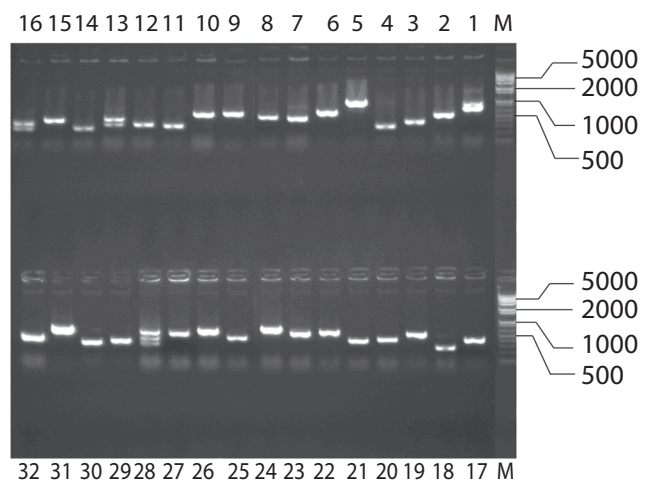

Fig. 1. PCR amplification of positive clones randomly selected from the forward and reversed libraries. M: $1 \mathrm{kbp}$ plus dna ladder (invitrogen), 1-32: PCR products of positive clones selected from forward (A) and reversed (B) libraries. 
TABLE 3

Partial genes whose number of ESTs determined in our experiment through BlastX was larger than 2 in the forward subtraction library

\begin{tabular}{lll}
\multicolumn{1}{c}{ Gene annotation } & \multicolumn{1}{c}{ Accession No. of best hit } & \multicolumn{1}{c}{ Species } \\
Similar to Adenosylhomocysteinase-like protein & NP_001067845.2 & Oryza sativa \\
rRNA intron-encoded homing endonuclease & XP_003614387.1 & Medicago truncatula \\
Mitochondrial protein, putative & XP_003588355.1 & Medicago truncatula \\
ATP-dependent zinc metalloprotease FTSH 5, mitochondrial-like & XP_003569240.1 & Brachypodium distachyon \\
predicted protein & XP_002328114.1 & Populus trichocarpa \\
ZIP transporter & XP_002315981.1 & Populus trichocarpa \\
with no lysine kinase 12 & NP_001236036.1 & Glycine max \\
triose phosphate isomerase cytosolic isoform & ACF06445.1 & Elaeis guineensis \\
Multidrug resistance protein ABC transporter family & XP_003607667.1 & Medicago truncatula \\
homeobox-leucine zipper protein HOX16-like & XP_003572981.1 & Brachypodium distachyon \\
putative mitochondrial 2-oxoglutarate/malate carrier protein-like & XP_003531254.1 & Glycine max \\
IAA9 & AEV89263.1 & Musa acuminata \\
hypothetical protein & XP_002464109.1 & Sorghum bicolor \\
Pathogenesis-related protein PRB1-2 & P35792.1 & Hordeum vulgare \\
hypothetical protein & XP_002567556.1 & Penicillium chrysogenum \\
phenylalanine ammonia-lyase & BAG70992.1 & Musa balbisiana \\
pathogenesis-related protein & AEV89264.1 & Musa acuminata \\
hypothetical protein & XP_002464109.1 & Sorghum bicolor \\
Rb7 & AAB53329.1 & Solanum lycopersicum \\
S-adenosylmethionine synthase & O22338.1 & Musa acuminata \\
hypothetical protein, partial & XP_003614388.1 & Medicago truncatula \\
uncharacterized protein & XP_003572828.1 & Brachypodium distachyon \\
putative glycosyltransferase & NP_001105849.1 & Zea mays \\
\hline & &
\end{tabular}

clones for reversed subtraction. Among them, 1000 clones were randomly selected from each library for sequencing. In order to estimate the rate of recombination and the length of the inserted fragment, we randomly selected 32 colonies each from the forward and reversed subtraction libraries for PCR. As shown by the electrophoresis results (Fig. 1A and 1B), the recombination rate of the library was above $95 \%$, and the sizes of insert fragments were 300-600bp.

ESTs assembly: After preprocessing, ESTs clustering and ESTs assembly, 737 sequences (527 unigenes) from the forward library and 757 sequences (483 unigenes) from the reversed library were considered as informative ESTs. There were 75 contigs and 472 singletons in forward unigenes, and there were 105 contigs and 378 singletons in reversed unigenes. The average length of all these unigenes were $479 \mathrm{bp}$. The longest unigene had $2064 \mathrm{bp}$, and the shortest unigene had 100bp.

BlastX analysis: Our BlastX search against the NR database showed that 522 unigenes in the forward library and 479 unigenes in the reversed library had homologous sequences in GenBank. Table 3 (forward library) and Table 4 (reversed library) list the information of unigenes that had more than 2 ESTs in our experiment. Comparing these two tables, we could see there were more transporter proteins, such as ZIP transporter, multidrug-resistance protein ATP-binding cassette (ABC) transporter family, and putative mitochondrial 2-oxoglutarate/ malate carrier protein in E. crassipes roots cultured under nitrogen deficient condition, suggesting that nitrogen transport may be related to these transporters. 
TABLE 4

Partial genes whose number of ESTs determined in our experiment through BlastX was larger than 2 in the reverse subtraction library

\begin{tabular}{lll}
\multicolumn{1}{c}{ Gene annotation } & \multicolumn{1}{c}{ Accession No. of best hit } & \multicolumn{1}{c}{ Species } \\
ATP-dependent zinc metalloprotease FTSH 5, mitochondrial-like & XP_003569240.1 & Brachypodium distachyon \\
hypothetical protein, malate dehydrogenase & XP_002558886.1 & Penicillium chrysogenum \\
hypothetical protein & NP_001067845.2 & Oryza sativa \\
with no lysine kinase 12 & NP_001236036.1 & Glycine max \\
e3 ubiquitin-protein ligase UBR4-like & XP_002915692.1 & Ailuropoda melanoleuca \\
hypothetical protein & XP_002567556.1 & Penicillium chrysogenum \\
actin, alpha cardiac muscle 1-like isoform 2 & XP_003442992.1 & Oreochromis niloticus \\
translationally controlled tumor protein & ACF06596.1 & Elaeis guineensis \\
hypothetical protein & XP_002559930.1 & Penicillium chrysogenum \\
fasciclin-like arabinogalactan protein 8-like & XP_003580362.1 & Brachypodium distachyon \\
putative glycosyltransferase & NP_001105849.1 & Zea mays \\
NAC protein 1 & AEQ94175.1 & Elaeis guineensis \\
uncharacterized protein & XP_003572828.1 & Brachypodium distachyon \\
rRNA intron-encoded homing endonuclease & XP_003614387.1 & Medicago truncatula \\
tubulin alpha-1 chain-like, partial & XP_003635553.1 & Vitis vinifera \\
Mitochondrial protein, putative & XP_003588355.1 & Medicago truncatula \\
DC24 & AAG44628.1; AF253979_1 & Homo sapiens \\
uncharacterized protein & XP_003631610.1 & Vitis vinifera \\
histone 2 & refXP_002307619.1 & Populus trichocarpa
\end{tabular}

In addition, adenosylhomocysteinase-like protein was also more abundant in nitrogen deficient E. crassipes. In those cultured in nitrogen replete medium, there were more proteins such as UBR4-like e3 ubiquitin-protein ligase and fasciclin-like arabinogalactan protein 8-like, as well as more cytoskeletal components, such as actin and tubulin.

COG analysis: After a homology search against the COG database, 195 and 148 ESTs in the forward and reversed libraries were identified to have best hits. These COG-informative ESTs were classified into several clusters according to their functions (Fig. 2A and 2B). In the forward library, ESTs involved in coenzyme transporter and metabolism (45 ESTs) were the most abundant, followed by ESTs participating in metabolism (37 ESTs). In the reversed library, cytoskeletal ESTs (22 ESTs) accounted for the largest number, followed by ESTs participating in information storage and processing (20 ESTs).
GO analysis: In GO analysis, all functions are clustered into three parts: cellular components, molecular functions, and biological processes. Overall, 239 and 183 unigenes in the forward and reversed libraries had GO information, respectively. Their categories are shown in Fig. 3. Unigenes related to binding and metabolic process were the most, followed by unigenes related to catalytic and cellular process. The most differentially expressed unigenes between forward and reversed libraries were those involved in binding, cellular process and electron carrier. The numbers of forward and reversed unigenes in these categories were 140 and 90, 109 and 66, 13 and 2 respectively. Their p-values were all below the significant level of 0.05 indicated remarkable relationship between the number of forward and reversed unigenes.

qRT-PCR analysis of differential unigenes: The results of qRT-PCR of ESTs are listed in Fig. 4. With regard to the forward 


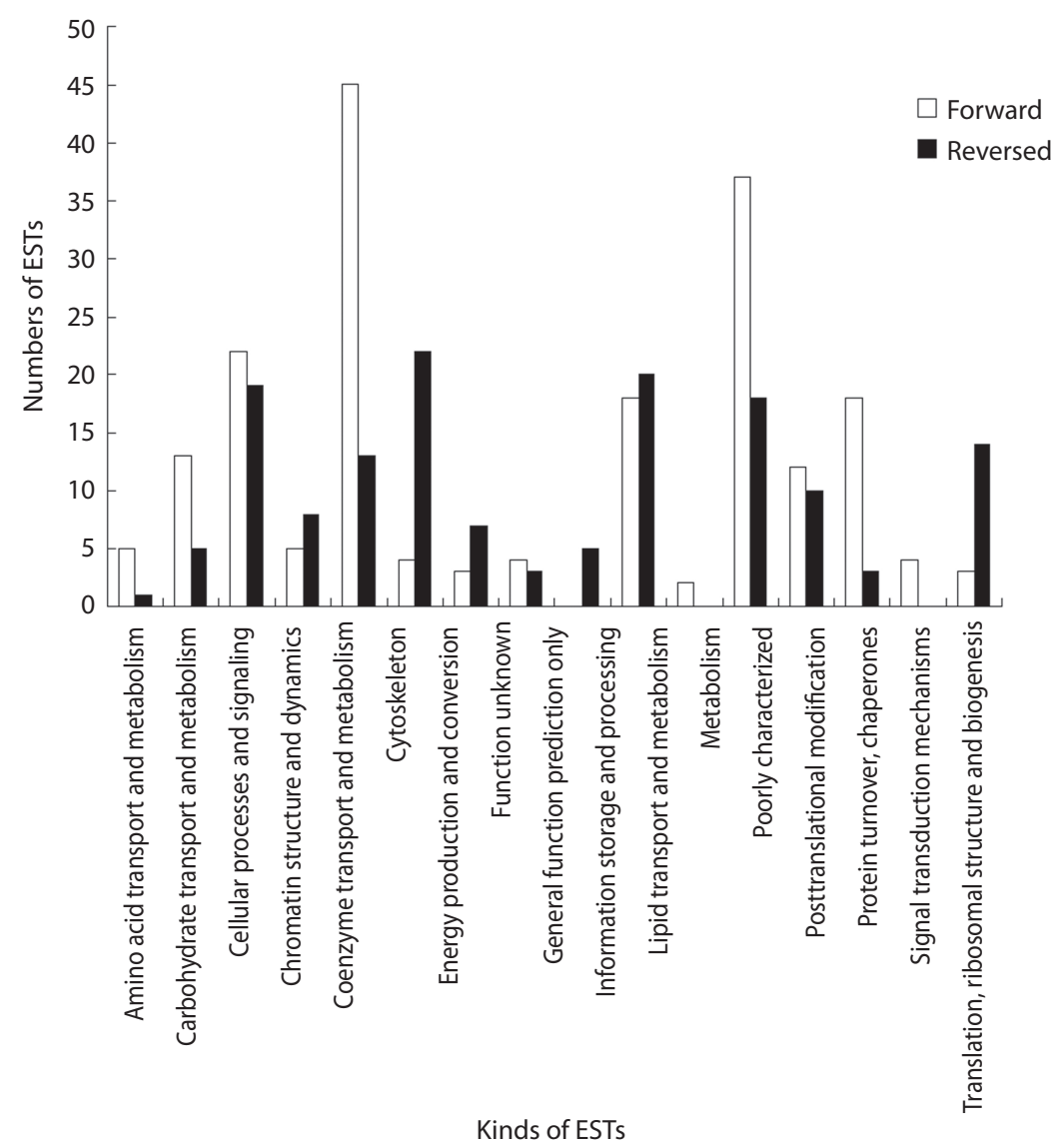

Fig. 2. Classification of all COG informative EST sequences from the forward and reversed subtraction libraries. E-value threshold was set to $10^{-10}$.

library selections, the expression levels of all 10 unigenes in samples cultured in nitrogen deficient medium were higher than those from samples cultured in replete nitrogen. These 10 unigenes were selected from the forward library. For the reversed library selections, the expression levels of 10 out of 11 unigenes from samples cultured in nitrogen deficient medium were lower than that from E. crassipes roots cultured in replete; unigene 19 was the exception. These 11 unigenes were selected from reversed library. This result further confirmed the $\mathrm{SSH}$ construction.

\section{DISCUSSION}

Aquatic plants play important roles in water ecological system, and water environment can also influence the physiology and ecology of aquatic plants. Previous reports have demonstrated that aquatic plants could be used efficiently in phytoremediation of eutrophic water (Casabianca et al., 1995; El-Gendy et al., 2006; Lu et al., 2008; Chen et al., 2010; Wang et al., 2013), while water eutrophication was also closely related to algae blooms, as well as the decline and disappearance of 


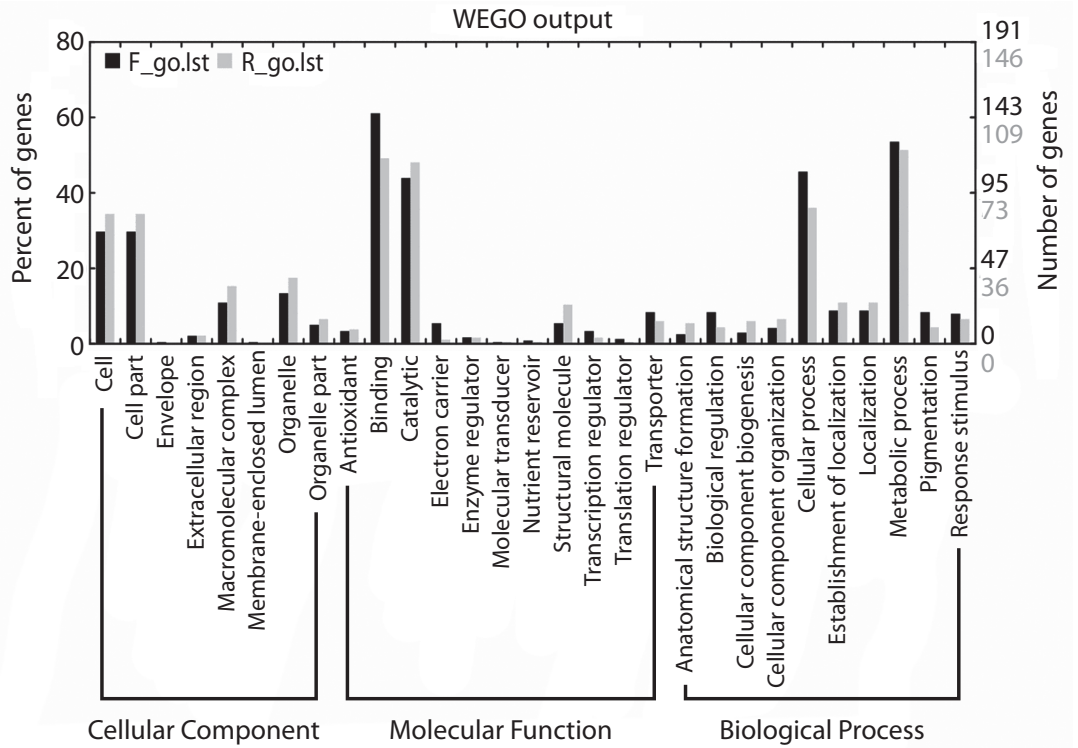

Fig. 3. Classification of all GO informative unigenes from the forward and reverse subtraction libraries on gene ontology. E-value threshold was set to $10^{-5}$.

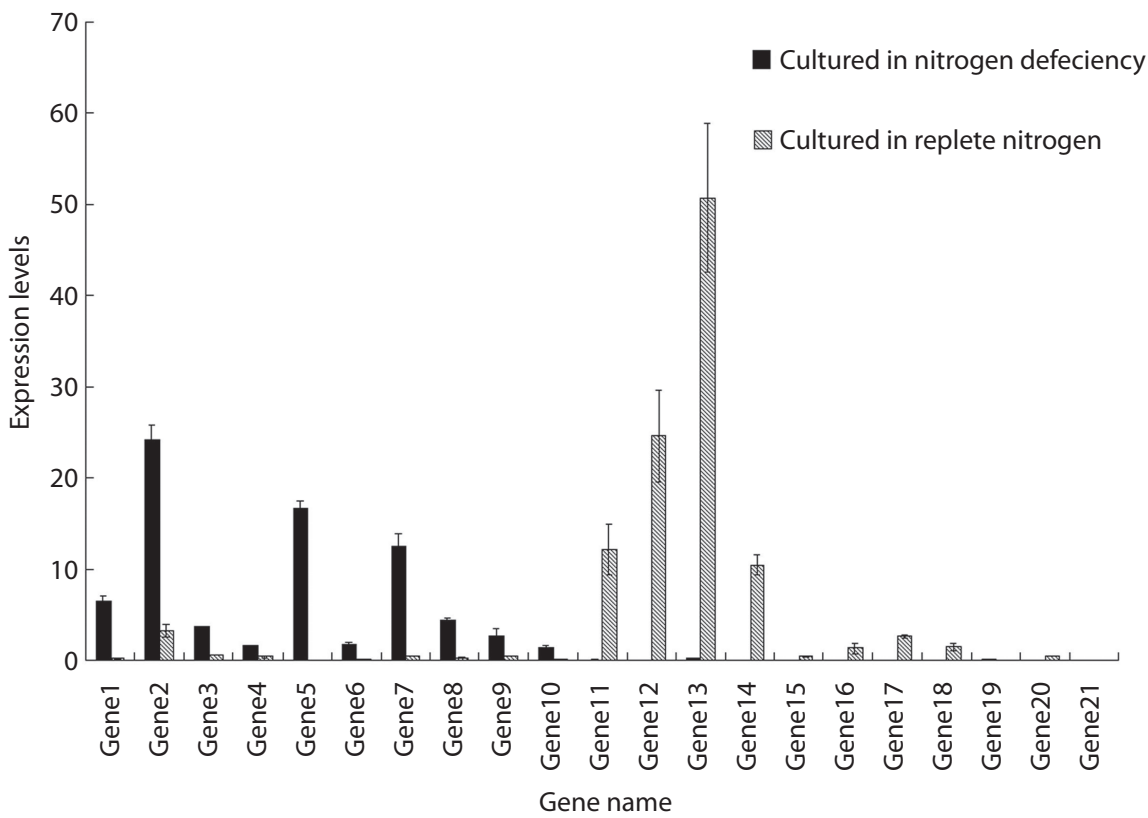

Fig. 4. qRT-PCR analysis of differential unigenes responsive to different nitrogen conditions. Samples were analyzed in triplicate. Data represent mean values, normalized with E. crassipes actin gene, and the error bars indicate the range of the replicate values. 
submerged plants and uncontrollable increases in E. crassipes population (Dyhrman, 2008; Ripley et al., 2006; Wang, Zhang, Wang, Li, \& $\mathrm{Lu}, 2010)$. The complexity of nutrient loading is matched by the complexity of nutritional strategies and capabilities of aquatic plants. Intensive study regarding the response at transcriptional level of aquatic plants to the status of different nutrients in water can help to elucidate the molecular mechanisms of the interaction between water environment and aquatic plants, and provide a theoretical basis for further develop aquatic plants in phytoremediation of eutrophic water. We can also use these differential expression genes as targets to regulate the physiology and ecology of aquatic plant from nutrient metabolism perspective in order to protect the balance of water ecology (Flores \& Herrero, 2005; Dyhrman, 2008; Wurch, Haley, Orchard, Gobler, \& Dyhrman, 2011).

Currently, research into the molecular mechanisms for aquatic plants to respond to nutrients in the environment has mainly focused on lower aquatic plants that could cause algae blooms. Wurch et al. (2011) used Long-SAGE (serial analysis of gene expression) to profile the transcriptome of the brown tide-forming algae Aureococcus anophagefferens and identified a suite of genes upregulated by nitrogen and phosphorus deficiency. Genes upregulated under nitrogen deficiency included an ammonium transporter, an acetamidase/formamidase, and two peptidases. Hildebrand (2005) also found an ammonium transporter expressed highly in nitrogen deficiency cell from the diatom Cylindrotheca fusiformis. Berg, Shrager, Glöckner, Arrigo, \& Grossman (2008) found that in the algae $A$. anophagefferens, growth in ammonium solution resulted in an increase in the relative expression of an ammonium transporter, a novel $\mathrm{ABC}$ transporter, and a putative high-affinity phosphate transporter. Nitrogen limitation resulted in a 30- to 110 -fold increase in the relative expression of nitrate, ammonium, urea, amino acid/polyamine, and formate/nitrite transporters.

In our experiment, there were also many transporters including ZIP transporter, multidrug-resistance protein $\mathrm{ABC}$ transporter family, and putative mitochondrial 2-oxoglutarate/malate carrier protein in nitrogen-deficient E. crassipes. This was consistent with the reports described above. The 2-oxoglutarate/ malate carrier protein can transport 2-oxoglutarate into the cytoplasm, where it may be involved in the process of nitrogen incorporation into carbon skeletons through the glutamine synthetase-glutamate synthase cycle, in which 2-oxoglutarate is the final nitrogen acceptor. Another function of 2-oxoglutarate is its role in nitrogen control. In cyanobacteria, the expression of many nitrogen-assimilation genes is subjected to regulation being activated by the nitrogen-control transcription factor NtcA, which is autoregulatory and whose activity appears to be influenced by 2-oxoglutarate and the signal transduction protein P(II) (Flores \& Herrero, 2005; Herrero, Muro-Pastor, \& Flores, 2001). In E. crassipes, whether 2-oxoglutarate is involved in nitrogen control still needs further study. ABC transporters utilize the energy of ATP hydrolysis to transport various substrates across cellular membranes. That nitrogen deficiency could increase the expression of $\mathrm{ABC}$ transporter gene suggests that this transporter might be involved in the transportation of nitrogen in E. crassipes. ZIP transporter can transport heavy metals such as Fe, Zn, Mn, $\mathrm{Cu}, \mathrm{Cd}$ across the membrane. Why could nitrogen deficiency increase the expression of this gene in E. crassipes? Could it also transport ammonium into the cell or is there any connection between transportation of ammonium and heavy metals so that nitrogen deficiency can influence the expression of the ZIP transporter? Further study should solve this problem. Besides this, we also identified a novel protein adenosylhomocysteinase-like protein expressed at higher levels in nitrogen deficient E. crassipes. This protein is the key enzyme involved in the methylation reaction, indicating that nitrogen assimilation and utilization may be related to the process of methylation in E. crassipes. We did not identify increased ammonium transporters expression in nitrogendeficient E. crassipes; perhaps ammonium 
can be indirectly transported through other transporters (Bertl, Reid, Sentenac, \& Slayman, 1997; Liu, Ludewig, Gassert, Frommer, \& von Wirén, 2003; Loqué, Ludewig, Yuan, \& von Wirén, 2005; Jahna et al., 2004). Another possible reason for this was that the differential expression of the ammonium transporters in $E$. crassipes was very low and was undetectable with the methods used here.

However, we found that UBR4-like e3 ubiquitin-protein ligase, fasciclin-like arabinogalactan protein 8-like, and some cytoskeletal protein such as actin and tubulin were upregulated in E. crassipes cultured in ammonium solution. E3 ubiquitin-protein ligase has many functions: it is a component of the $\mathrm{N}$-end rule pathway, it forms meshwork structures involved in membrane morphogenesis and cytoskeletal organization together with clathrin, and regulates integrin-mediated signaling (Nakatani et al., 2005). Fasciclin-like arabinogalactan protein 8-like is a cell-surface adhesion protein and may be significant in the process of competence acquisition (Johnson, Johes, Bacic, \& Schultz, 2003). Perhaps ammonium can efficiently improve certain metabolism processes, and enhance specific signal transduction pathways in E. crassipes. But which metabolic processes and which signal pathway could be influenced by ammonium in E. crassipes needs to be studied. It is also possible that higher levels of ammonium nitrogen allow E. crassipes to resist increases in osmotic pressure, partially due to alterations in cytoskeletal construction.

In conclusion, the function analysis of ESTs related to nitrogen deficiency and replete nitrogen in E. crassipes suggested that $E$. crassipes could respond to different nitrogen status by efficiently regulating and controlling some transporter gene expressions, certain metabolism processes, specific signal transduction pathways and cytoskeletal construction.

\section{ACKNOWLEDGMENT}

This work was funded by the Chinese National Science Foundation (grant 21177029) and a grant from the 211 project of Guangdong University of Technology.

\section{RESUMEN}

Identificación de fragmentos de genes relacionados con la deficiencia de nitrógeno en Eichhornia crassipes (Pontederiaceae). Se ha convertido en una maleza importante en hábitats de agua dulce en ríos, lagos y embalses, tanto en zonas tropicales como templadas de todo el mundo. Algunas investigaciones han indicado que se puede utilizar para la fitorremediación de agua, debido a su fuerte asimilación de nitrógeno y fósforo, y la acumulación de metales pesados, su crecimiento y propagación puede desempeñar un papel importante en la ecología ambiental. Con el fin de explorar el mecanismo molecular de respuesta a la deficiencia de nitrógeno en E. crassipes, se construyeron bibliotecas de cDNA mediante síntesis adelantada y retrasada para raíces de E. crassipes en condiciones de deficiencia de nitrógeno mediante el método de hibridación supresiva sustractiva (SSH). Para este estudio se utilizaron 2100 clones de síntesis adelantada y 2650 de síntesis retrasada. De la biblioteca se escogieron al azar mil clones, 737 (527 unigenes) de síntesis adelantada y 757 (483 unigenes) de síntesis retrasada que fueron informativos. El análisis BLASTX mostró que había más transportadores y proteínas adenosilhomocisteinasa en $E$. crassipes cultivadas en un medio deficiente de nitrógeno; mientras que las cultivadas en un medio repleto de nitrógeno tenían más proteínas como UBR4 e3 ubiquitina-proteína ligasa y la proteína arabinogalactano 8 tipo fasciclina, así como otras proteínas del citoesqueleto, incluyendo la actina y la tubulina. Clúster del Grupo Ortológico (COG) también demostró que en la biblioteca de síntesis adelantada, la mayoría de los marcadores de secuencia expresada (ESTs) estaban involucrados en el transporte de coenzimas y el metabolismo.

Palabras clave: Eichhornia crassipes, SSH, deficiencia de nitrógeno, análisis BlastX, análisis COG, análisis GO.

\section{REFERENCES}

Altschul, S. F., Gish, W., Miller, W., Myers, E. W., \& Lipman, D. J. (1990). Basic local alignment search tool. Journal of Molecular Biology, 215, 403-410.

Berg, G. M., Shrager, J., Glöckner, G., Arrigo, K. R., \& Grossman, A. R. (2008). Understanding nitrogen limitation Aureococcus anophagefferens (Pelagophyceae) through cDNA and qRT-PCR analysis. Journal of Phycology, 44, 1235-1249.

Bertl, A., Reid, J. D., Sentenac, H., \& Slayman, C. L. (1997). Functional comparison of plant inward rectifier channels expressed in yeast. Journal of Experimental Botany, 48, 405-413. 
Caldelas, C., Iglesia-Turino, S., Araus, J. L., Bort, J., \& Febrero, A. (2009). Physiological responses of Eichhornia crassipes (Mart.) Solms to the combined exposure to excess nutrients and Hg. Brazilian Journal of Plant Physiology, 21, 01-12.

Casabianca, M. L. D., Laugier, T., \& Posada, F. (1995). Petroliferous wastewater treatment with water hyacinth: experimental statement. Waste Management, $15,651-655$.

Chen, X., Chen, X. X., Wan, X. W., Weng, B. Q., \& Huang, Q. (2010). Water hyacinth (Eichhornia crassipes) waste as an adsorbent for phosphorus removal from swine wastewater. Bioresource Technology, 101, 9025-9030.

Deng, H., Ye, Z. H., \& Wong, M. H. (2004). Accumulation of lead, zinc, copper and cadmium by 12 wetland plant species thriving in metal-contaminated sites in China. Environmental Pollution, 132, 29-40.

Diatchenko, L., Lau, Y. F. C., Campbell, A. P., Chenchik, A., Moqadam, F., Huang, B., Lukyanov, S., Lukyanov, K., Gurskaya, N., Sverdlov, E. D., \& Siebert, P. D. (1996). Suppression subtractive hybridization: A method for generating differentially regulated or tissue-specific cDNA probes and libraries. Proceedings of the National Academy of Sciences of the Unite States of America, 93, 6025-6030.

Dyhrman, S. T. (2008). Molecular approaches to diagnosing nutritional physiology in harmful algae: Implications for studying the effects of eutrophication. Harmful Algae, 8, 167-174.

El-Gendy, A. S., Biswas, N., \& Bewtra, J. K. (2006). Municipal landfill leachate treatment for metal removal using water hyacinth in a floating aquatic system. Water Environment Research, 78(9), 951-964.

Flores, E. \& Herrero, A. (2005). Nitrogen assimilation and nitrogen control in cyanobacteria. Biochemical Society Transactions, 33, 164-166.

Herrero, A., Muro-Pastor, A. M., \& Flores, E. (2001). Nitrogen control in cyanobacteria. Journal of Bacteriology, 183, 411-425.

Hildebrand, M. (2005). Cloning and functional characterization of ammonium transporters from the marine diatom Cylindrotheca fusiformis. Journal of Phycology, 41, 105-113.

Jahna, T. P., Anders, L. B., Zeuthen, T., Holm, L. M., Klaerke, D. A., Mohsin, B., Kühlbrandt, W., \& Schjoerring, J. K. (2004). Aquaporin homologuesin plants and mammals transport anunonia. FEBS Letters, 574, 31-36.

Johnson, K. L., Johes, B. J., Bacic, A., \& Schultz, C. J. (2003). The fasciclin-like arabinogalactan proteins of Arabidopsis. A multigene family of putative cell adhesion molecules. Plant Physiology, 133, 1911-1925.
Liu, L. H., Ludewig, U., Gassert, B., Frommer, W. B., \& von Wirén, N. (2003). Urea transport by nitrogenregulated tonoplast intrinsic proteins in Arabidopsis. Plant Physiology, 133, 1220-1228.

Livak, K. J. \& Schmittgen, T. D. (2001). Analysis of relative gene expression data using real-time quantitative PCR and the 2(-Delta Delta C(T)) method. Methods, $25,402-408$.

Li, W. G., Gong, H. M., \& Chang, T. J. (2008). Efects of nitrogen form on growth and physiological responses of an aquatic plant Eichhornia crassipes. Journal of Agro-Environment Science, 27, 1545-1549.

Loqué, D., Ludewig, U., Yuan, L. X., \& von Wirén, N. (2005). Tonoplast intrinsic proteins AtT1P2;1 and AtT1P2;3 facilitate NH3 transport into the vacuole. Plant Phlysiology, 137, 671-680.

Lu, J. B., Fu, Z. H., \& Yin, Z. Z. (2008). Performance of a water hyacinth (Eichhornia crassipes) system in the treatment of wastewater from a duck farm and the effects of using water hyacinth as duck feed. Journal of Environmental Sciences, 20, 513-519.

Nakatani, Y., Konishi, H., Vassilev, A., Kurooka, H., Ishiguro, K., Sawada, J., Ikura, T., Korsmeyer, S. J., Qin, J., \& Herlitz, A. M. (2005). p600, a unique protein required for membrane morphogenesis and cell survival. Proceedings of the National Academy of Sciences of the Unite States of America, 102, 15093-15098.

Odjegba, V. J. \& Fasidi, I. O. (2007). Changes in antioxidant enzyme activities in Eichhornia crassipes (Pontederiaceae) and Pistia stratiotes (Araceae) under heavy metal stress. Revista de Biología Tropical, 55, 815-823.

Reddy, K. R., Agami, M., \& Tucker, J. C. (1989). Influence of nitrogen supply rates on growth and nutrient storage by water hyacinth (Eichhornia crassipes (Mart.) Solms) plants. Aquatic Botany, 36, 33-43.

Reddy, K. R., Agami, M., \& Tucker, J. C. (1990). Influence of phosphorus supply rates on growth and nutrient storage by water hyacinth (Eichhornia crassipes (Mart.) Solms) plants. Aquatic Botany, 37, 355-365.

Ripley, B. S., Muller, E., Behenna, M., Whittington-Jones, G. M., \& Hill, M. P. (2006). Biomass and photosynthetic productivity of water hyacinth (Eichhornia crassipes) as affected by nutrient supply and mirid (Eccritotarus catarinensis) biocontrol. Biological Control, 39, 392-400.

Tatusov, R. L., Koonin, E. V., \& Lipman, D. J. (1997). A genomic perspective on protein families. Science, 278, 631-637.

Tatusov, R. L., Fedorova, N. D., Jackson, J. D., Jacobs, A. R., Kiryutin, B., Koonin, E. V., Krylov, D. M., Mazumder, R., Mekhedov, S. L., Nikolskaya, A. N., Rao, B. S., Smirnov, S., Sverdlov, A. V., Vasudevan, S., Wolf, Y. I., Yin, J. J., \& Natale, D. A. (2003). The 
COG database: an updated version includes eukaryotes. BMC Bioinformatics, 4, 41.

Wang, C., Zhang, S. H., Wang, P. F., Li, W., \& Lu, J. (2010). Effects of ammonium on the antioxidative response in Hydrilla verticillata (L.f.) Royle plants. Ecotoxicology and Environmental Safety, 73, 189-195.

Wang, Z., Zhang, Z., Zhang, Y., Zhang, J., Yan, S., \& Guo, J. (2013). Nitrogen removal from Lake Caohai, a typical ultra-eutrophic lake in China with large scale confined growth of Eichhornia crassipes. Chemosphere, 92(2), 177-83. doi: 10.1016/j. chemosphere.2013.03.014
Wurch, L. L., Haley, S. T., Orchard, E. D., Gobler, C. J., \& Dyhrman, S. T. (2011). Nutrient-regulated transcriptional responses in the brown tide-forming alga Aureococcus anophagefferens. Environmental Microbiology, 13, 468-481.

Xie, Y. H., Wen, M. Z., Yu, D., \& Li, Y. K. (2004). Growth and resource allocation of water hyacinth as affected by gradually increasing nutrient concentrations. Aquatic Botany, 79, 257-266.

Ye, J., Fang, L., Zheng, H., Zhang, Y., Chen, J., Zhang, Z., Wang, J., Li, S., Li, R., Bolund, L., \& Wang, J. (2006). WEGO: a web tool for plotting GO annotations. Nucleic Acids Research, 34, W293-7. 